\title{
DESIGN E TECNOLOGIA: EXPERIÊNCIA DE FORMAÇÃO DE UM GRUPO DE ESTUDOS EM PROTOTIPAGEM ELETRÔNICA POR DESIGNERS
}

\author{
Katarine Inis Soares Santos, acadêmica \\ Universidade do Estado de Minas Gerais \\ katarineinis@gmail.com \\ Matheus de Souza Silva, acadêmico \\ Universidade do Estado de Minas Gerais \\ souzamatheus93@gmail.com \\ Hebert Phillipe Martins Pereira, acadêmico \\ Faculdade de Comunicação e Artes, PUC Minas \\ hebert.pereira@sga.pucminas.br \\ Pedro de Oliveira Brandão, acadêmico \\ Universidade do Estado de Minas Gerais \\ pbrandao.mg@gmail.com
}

Resumo: O Movimento Maker é um dos fatores responsáveis pela democratização dos meios de produção. Por meio do contato com novas tecnologias de prototipagem, o designer se encontra agora em um amplo campo de atuação que, anteriormente, era restrito à indústria. O objetivo desse artigo é descrever o surgimento do grupo de pesquisa em Sistemas Interativos "Arduemgers", sua relação com o movimento Maker e os workshops como meio de aprendizagem. São analisados a história do movimento Maker, o método didático do Critical Making e sua aplicação como processo projetual. Por fim, são analisadas as contribuições dos projetos e dos eventos realizados pelo grupo que buscam aproximar a comunidade acadêmica desta nova ferramenta criativa.

Palavras-chave: Critical Making, Prototipagem Eletrônica, Movimento Maker, Grupo de Estudos, Makerspaces

Abstract: The Maker Movement is one of the factors responsible for the democratization of the means of production. Through the contact with new prototyping technologies, the designer is now in a wide acting field that previously was restricted to the industry. The aim of this article is to describe the foundation of the interactive systems research group "Arduemgers", its relationship with the Maker movement and the workshops as a learning method. The authors analyzed the history of the Maker movement, the Critical Making teaching method and its application as a design process. Finally, the contributions of projects and events held by 
the group are analyzed, seeking to approximate the academic community to this new creative tool.

KEYWORDS: Critica Making, Electronic Prototyping, Maker Movement, Study Group, Makerspaces

\section{INTRODUÇÃO}

O Grupo Arduemgers surgiu de uma iniciativa dos alunos da Escola de Design (BH) da Universidade do Estado de Minas Gerais. Em agosto de 2015, por meio de um post de Facebook no grupo da escola, alguns alunos decidiram se reunir para aprenderem juntos a utilizar o Arduíno como plataforma de prototipagem de projetos de design. Ao observar a movimentação na rede social, o Prof. MSc André Carvalho Mol Silva, coordenador do Centro Design Empresa (CDE), ofereceu o apoio do centro com espaço e compra de equipamentos para o início das atividades. Desde então esta tem sido a base de funcionamento do grupo de pesquisa em Sistemas Interativos, "Arduemgers". O grupo realiza dois encontros semanais, e desde fevereiro de 2016 conta com a orientação da Profa Dra. Fernanda da Costa Portugal Duarte.

O grupo, após alguns meses de experimentações, transferiu o foco dos projetos da tecnologia para as possíveis aplicações, ou seja, a prototipagem passou a ser uma ferramenta de aprimoramento de habilidades que serviriam de suporte técnico ao trabalho conceitual. Ratto (2011) apresenta o termo Critical Making como uma forma de associação de duas formas de engajamento do mundo: teórica e prática, ou conceitual e material. A pesquisa extensiva de uma literatura; seleção de conceitos e ideias; fabricação de protótipos, bem a exploração de diversas configurações com o objetivo de alcançar máxima expressão, são processos interdependentes que os sete membros frequentes buscam aplicar nos projetos. Para tanto as reuniões semanais são organizadas da seguinte forma: às terças são discutidos artigos ou livros que servem de aporte teórico para as atividades prática a serem executadas aos sábados.

O Arduíno é uma plataforma de prototipagem eletrônica com hardware e software "open source", ou seja, de "código aberto". O open source é uma prática de disponibilização das "configurações básicas" do software e hardware que permite que outras pessoas, além do criador, sejam capazes de aprimorar a tecnologia. Tem como princípio a ideia de cocriação, diferente do software proprietário que prima pela proteção intelectual (copyright). Foi projetado para que entusiastas fossem capazes de gerar soluções para problemas do dia a dia sem precisar de anos de estudos em uma escola de engenharia. Portanto, se trata de uma comunidade colaborativa mundial com inúmeras bibliotecas de projetos abertos a reprodução e aprimoramento. Os usuários do Arduíno, bem como de outras muitas plataformas, hoje integram um movimento em expansão, o Movimento Maker/DIY, ou "faça você mesmo".

Chris Anderson (2012, p.19) fala "the rise of "open hardware," another part of what's known as the Maker Movement, is now doing for physical goods what open source did for software ${ }^{1 "}$. Aqui o autor destaca o potencial dessa tecnologia em alterar

1 Tradução livre por autores: “o surgimento do "open hardware/hardware aberto", outra parte do que é conhecido como o Movimento Maker, está fazendo pelos bens físicos o que o open source fez pelo software." 
a forma como se ensina design, bem como a relação das pessoas com a própria criatividade. O open source não só representou uma mudança de comportamento dos desenvolvedores de software frente aos métodos de criação, mas impactou o próprio acesso às tecnologias pelos consumidores. A ascensão do open hardware, por sua vez, tende a democratizar a produção de objetos para o mundo físico quando torna o hardware mais acessível inclusive a não especialistas em eletrônica.

O fato de o grupo Arduemgers estar localizado no laboratório de prototipagem FAB LAB Design², parte do Centro Design Empresa (CDE), o torna privilegiado em termos de oportunidades de experimentação durante os projetos. O fab lab é um modelo de laboratório de fabricação que tem como princípio o fácil acesso de acadêmicos e comunidade a um espaço com equipamentos de prototipagem. Este laboratório somado às oficinas de madeira, metais e modelagem oferecem aos alunos a possibilidade de produção de artefatos de qualidade quase industrial. Assim ideias podem ser testadas com maior agilidade com baixo custo. A experiência de desenvolvimento de produtos ou serviços se torna completa quando a estrutura da escola e a atitude maker adotada pelo grupo criam ambiente criativo vívido.

Em abril de 2016 o grupo ofereceu a série de workshops à comunidade acadêmica da Escola de Design e de outras instituições de ensino superior. A série de workshops tinha como objetivo principal nivelar os conhecimentos técnico-conceituais dos participantes do Arduemgers, assim como atrair novos membros ao grupo. Além disso, com esta iniciativa, o grupo buscou aproximar relações com pesquisadores e estudantes de outras instituições.

Este artigo traz na primeira parte o Referencial Teórico em que são apresentadas a metodologia de pesquisa do grupo, bem como o fenômeno mundial ao que o Arduemgers integra; na parte denominada Workshop os autores apresentam como a série de workshops foi conduzida; nas Considerações Finais o grupo apresentou suas impressões sobre a experiência e indicou expectativas para o futuro do grupo, incluindo, a mudança na forma de atuação.

\section{REFERENCIAL TEÓRICO}

O Movimento Maker pode ser entendido como um fenômeno recente, caracterizado pelo envolvimento de hackers, estudantes, artistas, desenvolvedores e entusiastas de diversas áreas com a produção de objetos de uso, tanto para fins de recreação como para de desenvolvimento de produtos. A história do Movimento Maker, assim como a criação de seu nome, podem ser compreendidas a partir da fundação da revista Make, em 2005, e da realização da primeira Feira Maker, em 2006. Contudo, o processo de mobilizar técnicas e conhecimentos de produção para produzir objetos precede o Movimento Maker, estando presente em diversas atividades de transformação e manipulação de materiais realizadas por artistas, artesãos, e inventores ao longo da história (MARTIN; 2015). Apesar da iniciativa de cada projeto partir de um indivíduo ou de pequenos grupos, o Movimento Maker tem como uma de suas principais características o caráter colaborativo observado na maioria dos projetos. Nesta prática, denominada Open Source, os arquivos são disponibilizados

2 Fab Lab: do termo em inglês fabrication laboratory. Para mais informações: http://fab.cba.mit.edu/about/faq/. 
online para que outros makers possam fazer modificações, adaptações ou para que possam tentar reproduzir ou tentar construir o projeto disponibilizado.

Os indivíduos envolvidos no Movimento Maker se concentram em pequenas comunidades denominadas Makerspaces, onde participantes de diversas áreas e com variados níveis de conhecimento interagem em relação aos projetos desenvolvidos na comunidade. Os Makerspaces possuem espaços físicos destinados às atividades práticas dos participantes envolvidos, apresentando estrutura e equipamentos como computadores, impressoras 3D, componentes eletrônicos, máquinas de corte e usinagem CNC. Contudo, os Markerspaces não concentram apenas atividades de produção e desenvolvimento de projetos, mas também abrigam eventos como feiras, palestras, cursos, workshops e exposições a respeito dos trabalhos desenvolvidos e dos conhecimentos empregados para faze-los (HALVERSON, SHERIDAN; 2014).

As atividades práticas ligadas à produção de artefatos estão presentes em diversos momentos da história humana. Artesões, produtores, artistas e inventores estavam sempre relacionados às atividades e técnicas de produção de objetos artísticos e de função útil (CARDOSO; 2008). As técnicas de produção, criadas de forma empírica por artesãos, ou desenvolvidas por pesquisadores ou profissionais da indústria, ficavam restritas aos indivíduos que tinham acesso à determinado conhecimento por meio de suas atividades profissionais. Contudo, a facilidade de acesso às tecnologias de prototipagem rápida, juntamente com a disseminação de conhecimentos de produção e desenvolvimento de produtos e o aprimoramento de softwares de modelagem e simulação de projetos descentralizaram os meios de produção, inserindo entusiastas e desenvolvedores amadores em um ambiente anteriormente restrito a profissionais da indústria (BLIKSTEIN, KRANNICH; 2013).

Por outro lado, a evolução das tecnologias de produção industrial e a orientação do designer para projetos de produção em massa afastou a profissão da vocação histórica para a produção de artefatos. A nova organização do trabalho, implementada na revolução industrial, proporcionou uma divisão entre os profissionais responsáveis pela produção (operários) e o novo profissional da indústria, posteriormente conhecido como designer industrial, responsável pelo projeto dos novos produtos. Esta segmentação afastou o profissional de design dos processos de produção de produtos, limitando o contato do designer com os meios de produção aos estágios finais de desenvolvimento de um produto (BÜRDEK; 2010).

Esta mudança transformou o processo de produção, que ocorria paralelamente à concepção dos produtos e estava aberto às críticas do designer, em uma etapa posterior à criação do produto, direcionada para a avaliação e a validação do projeto. As etapas de experimentação são vitais para a construção de um processo criativo eficaz, pois a liberdade de exploração das possibilidades de projeto permite que o designer avalie um quadro amplo de soluções possíveis, aumentando o potencial de inovação do projeto desenvolvido. Além disso, as etapas de experimentação na produção de objetos incentivam o designer a refletir e criticar os processos adotados por ele em suas atividades, possibilitando a criação de novos meios para a concepção de objetos dento dos métodos de design.

A fim de diminuir o distanciamento existente entre os processos projetuais de design e as técnicas de produção, é possível mobilizar alguns aspectos fundamentais da cultura Maker. A experimentação está presente no Movimento Maker como suporte contínuo para os processos de aprendizado e concepção, criando uma conexão entre o 
planejamento de um projeto e a produção do objeto físico. Segundo o Manifesto do Movimento Maker (HATCH; 2013), a produção de objetos e artefatos é uma parte essencial da vida humana, e as atividades desempenhadas durante os processos de concepção estão embasadas nos princípios em diversos princípios básicos, entre eles a criação colaborativa e centrada no aprendizado.

A implementação dos conceitos do Movimento Maker é uma iniciativa que já é adotada para fomentar o interesse pela tecnologia entre estudantes do ensino básico. Através do contato com as novas tecnologias de produção, os educadores têm a expectativa de instigar o pensamento criativo das crianças, incentivando a apropriação dos meios tecnológicos para criação e o desenvolvimento de ideias que produzam valor no futuro (DOUGHERTY; 2013). Da mesma forma, este pensamento também pode ser usado para aproximar novamente o designer da experimentação crítica na produção de artefatos, oferecendo novos processos voltados para a inovação no ensino do design.

A fim de estabelecer novos processos capazes de produzir meios para a experimentação crítica no ensino do design, este trabalho adotou o método didático conhecido como Critical Making. Matt Ratto e a equipe do laboratório Critical Making da Universidade de Toronto, desenvolve a metodologia de critical making desde o início dos anos 2000. Esta metodologia de pesquisa trata de processos voltados para a exploração de formas de conexão com plataformas tecnológicas que fomentama reflexão e o pensamento crítico, conectando a relação entre indivíduo e tecnologia a experiências reais. Desta forma, o processo de pesquisa gera reflexões mais concretas sobre o processo de aprendizagem. O objetivo é combinar as etapas de pensamento crítico e experimentação prática, tendo objetos físicos como suporte para o processo (RATTO; 2011).

A metodologia de pesquisa proposta pelo Critical Making envolve abordagens multidisciplinares, centradas no processo de desenvolvimento do projeto. A primeira etapa descrita no método compreende uma revisão da literatura, destacando os principais conceitos e teorias relevantes para o objetivo da proposta de desenvolvimento. Esta etapa tem o objetivo de identificar princípios e conceitos contidos na literatura que possam ser transpostos para o ambiente físico através da fabricação e da prototipagem. Em um segundo momento, os atores envolvidos no processo partem para a construção de protótipos, explorando todas as áreas e os níveis de conhecimento disponíveis no grupo para geração de diversas soluções técnicas e conceituais possíveis. A última etapa envolve a reflexão coletiva dos atores a respeito dos resultados parciais, fazendo uso da crítica baseada nas duas etapas anteriores para a formulação de novos modelos, soluções e possibilidades (RATTO; 2011).

O objetivo do Critical Making é explicitar os resultados da ação conjunta dos atores envolvidos no ato de produção do projeto. Através do engajamento colaborativo dos participantes, é possível criar um processo de reflexão e crítica coletiva que ocorre simultaneamente à construção do artefato planejado. Os materiais manipulados durante o processo (componentes eletrônicos, peças mecânicas, computadores) são considerados elementos de transição, responsáveis por dar suporte físico ao pensamento construído de forma interativa pelo grupo. As reflexões desenvolvidas pelos participantes durante o processo são consideradas mais importantes do que o resultado final do artefato produzido, pois as conclusões 
extraídas do processo poderão servir como vetor para uma nova etapa de desenvolvimento (RATTO; 2011).

\section{WORKSHOP}

A fim de difundir o conhecimento através do Critical Making, os workshops se mostraram como a melhor ferramenta para a interação do público com as ferramentas da prototipagem eletrônica, aprofundando o envolvimento dos participantes com o processo de forma prática.

Os workshops abordaram a introdução de conceitos básicos de eletrônica e programação por meio do Arduíno, apresentando o movimento Maker ao público, em especial a comunidade acadêmica, sugerindo novas possibilidades de projetos e buscando desmistificar sua complexidade.

Foram feitos 4 workshops, nos dias 9 e 16 de abril e 7 e 14 de maio. No dia 9 de abril de 2016 aconteceu o primeiro workshop sobre lógica de programação. Nele foram abordados assuntos considerados base para o aprendizado da programação de sistemas, como: variáveis, tipagem, estruturas condicionais, estruturas de controle e repetição e funções. O público alvo esperado eram pessoas interessadas em aprender lógica de programação voltada para o microcontrolador Arduino. Os conceitos de lógica de programação foram introduzidos usando a linguagem de programação $C$, sendo esta a linguagem adotada pela plataforma Arduino, garantindo assim um maior aproveitamento do conteúdo ensinado ao termino do workshop.

Criada nos anos 70 por Dennis M. Ritchie (1993) no AT\&T Bell Labs para uso com o sistema operacional UNIX, a linguagem de programação $C$ se tornou muito popular com o tempo, sendo usada em diversas áreas. O C utilizado no Arduíno difere um pouco do $C$ criado por Dennis $M$. Ritchie, por ser necessário a implementação de duas funções: setup e loop, enquanto que em C, você basicamente só precisa da função main. Apesar disso, o conhecimento adquirido sobre a linguagem $C$ durante $o$ aprendizado da plataforma Arduíno pode ser aproveitado sem grandes dificuldades em outras plataformas ou linguagens, fazendo com que o Arduíno possa ser uma alternativa para interessados em aprender $\mathrm{C}$.

O Arduino utiliza um ambiente de desenvolvimento próprio, podendo ser baixado gratuitamente do site da empresa (Figura1). Esse ambiente permite que o código escrito possa ser testado e depois enviado para o microcontrolador, desde que este esteja conectado ao PC no qual o código está sendo enviado.

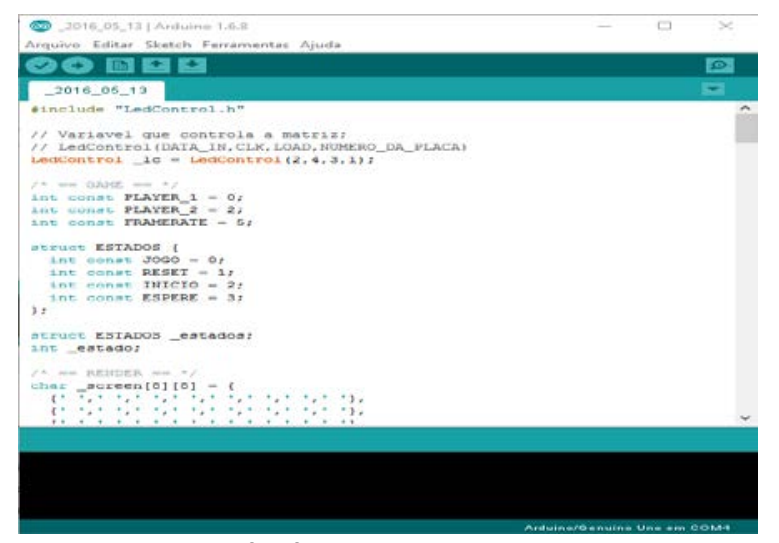

Figura 1: Exemplo de programa escrito em C.

Fonte: Projeto dos autores no ambiente de desenvolvimento do Arduíno. 
Para facilitar o aprendizado, foi usado método de ensino conhecido como "Analogia do post-it", que consiste em associar alguns elementos da programação com os papeis autoadesivos, tal como pode ser visto na figura 2. Também foram usados exercícios, tanto em sala quanto para se fazer em casa, como forma de fixar melhor o conhecimento adquirido.

\section{Variáveis}

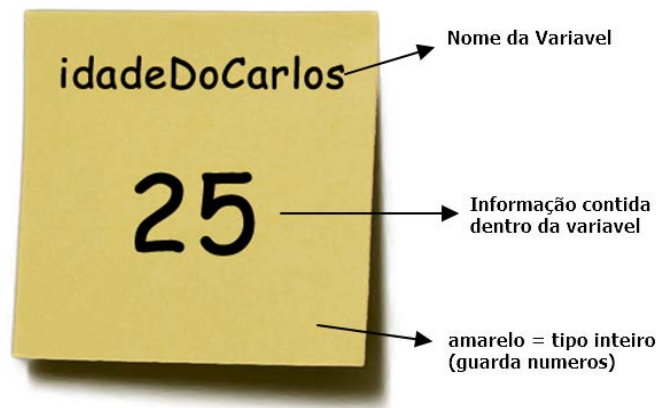

Figura 2: Exemplo de uso da "Analogia do post-it" no ensino de variáveis.

Fonte: Elaborado pelo autor, com base na pesquisa realizada.

No dia 16 de abril de 2016 ocorreu o segundo workshop de Arduíno lidando com a eletrônica na montagem do hardware de projetos. Por meio da analogia hidráulica, em que é feita uma correlação entre os circuitos elétricos, encanamento e água. Foram abordados os princípios básicos da elétrica, como o efeito Joule (relação entre corrente elétrica e calor) e a lei de Ohm (relação entre tensão, corrente e resistência), descrição, funcionamento e associação de componentes básicos eletrônicos. Foi apresentada a plataforma Arduíno, pelas versões Arduíno UNO R3 e Arduíno MEGA.

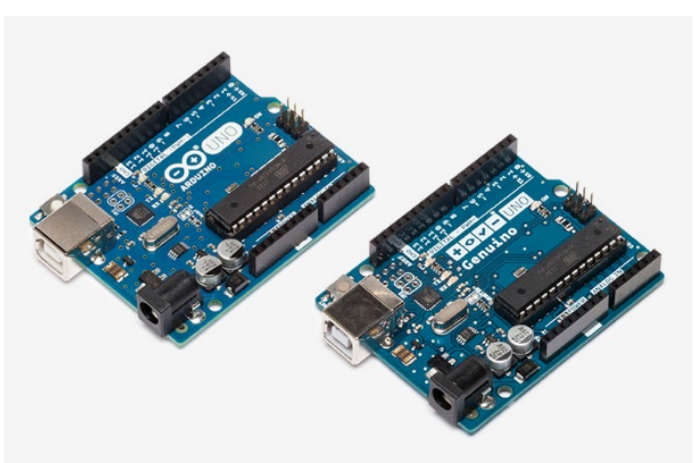

Figura 3: Arduino/Genuino UNO.

Fonte: https://www.arduino.cc/en/Main/ArduinoBoardUno

A plataforma Arduino, por ser open source no seu hardware, permite que sejam desenvolvidas várias versões compatíveis entre si. Existem 20 versões oficiais suportadas e inúmeras versões similares, com os mais variados ajustes e funções, como para vestíveis, robótica, internet das coisas e redes. Há também uma versão brasileira da placa, o Severino, desenvolvida por Adilson Akashi (Figura 4). Esta versão pode confeccionada pelo próprio usuário e tem suporte oficial (ARDUINO; 2016). 


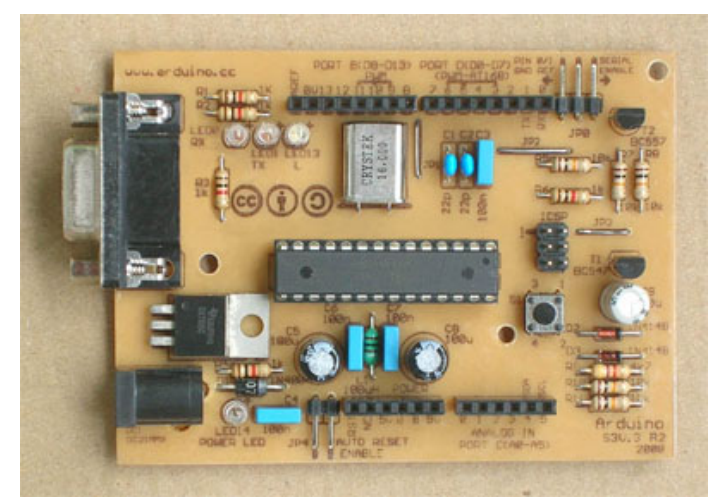

Figura 4: Severino versão 3.

Fonte: https://www.arduino.cc/en/Main/ArduinoBoardSerialSingleSided3

No dia 7 de maio foi realizado o terceiro workshop, onde pudemos unir o conteúdo ensinado nos dois últimos encontros e demonstrar como os conhecimentos ensinados trabalham no Arduíno. O público alvo esperado foram os alunos dos dois últimos workshops e demais pessoas interessadas em aprender Arduíno.

Usando o simulador 123D Circuits, iniciamos a aula ensinando como ligar um LED, transitando entre a parte eletrônica e a lógica, permitindo assim que os alunos pudessem compreender melhor como os dois conhecimentos eram utilizados no Arduino. Ao fim desse exercício, iniciamos o projeto final desse workshop: Um simulador de semáforo (Figura 5).

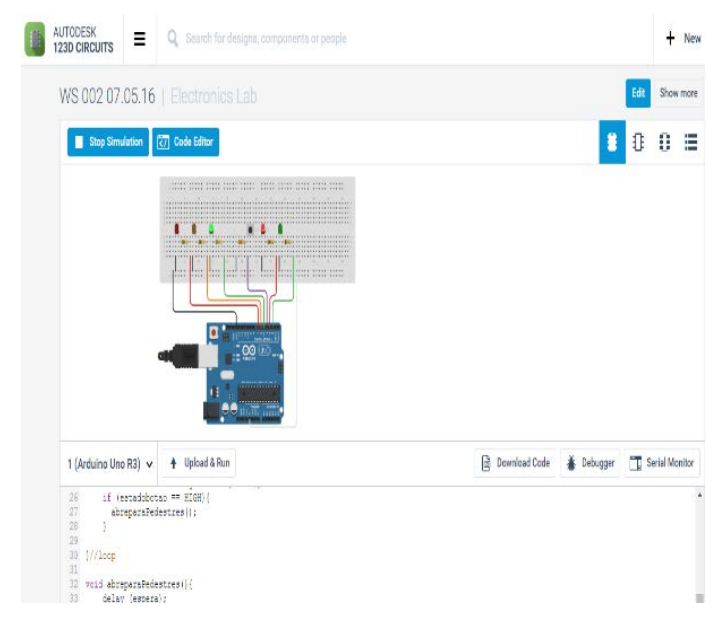

Figura 5: Recorte de tela do projeto semáforo usando o 123D Circuits.

Fonte: Projeto dos autores no simulador Autodesk 123D Circuits.

Na figura 5 podemos observar a interface do simulador em que desde a disposição e configuração dos componentes a eficácia do código podem ser verificados antes de testes reais. Dessa forma pequenos acidentes como a queima de componentes são evitados. Existem outros simuladores similares, pagos ou gratuitos, dentre eles o TINA. Optamos pelo 123D Circuits por ser uma plataforma online e gratuita dispensando o download da ferramenta.

Nesse projeto pudemos utilizar de forma mais ampla todos os conteúdos já apresentados nos workshops anteriores e pudemos introduzir novos, tanto na parte eletrônica quanto na parte lógica do trabalho. 
No dia 14 de maio aconteceu o quarto e último workshop, onde executamos o projeto do Pong, usando a plataforma Arduíno, juntamente com o simulador $123 \mathrm{D}$ Circuits. O Pong, um dos primeiros videogames bem sucedidos, foi desenvolvido, distribuído pela Atari e lançado em 29 de novembro 1972 como fliperama (Figura 6). 0 videogame simula um jogo de tênis de mesa de um ângulo panorâmico, onde cada jogador, ou o computador, controla uma raquete que rebate uma bola de volta para o jogador do outro lado. Os pontos são marcados quando a bola sai da tela no lado do jogador adversário (KENT; 2001).

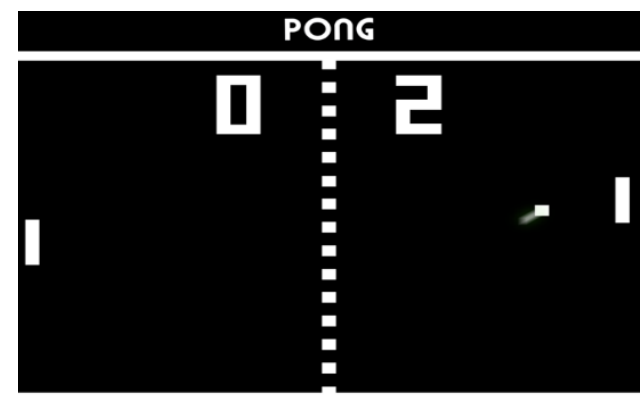

Figura 6: Imagem de tela do jogo PONG

Fonte: http://www.avantexperience.com/portfolio_chm.php

O projeto do Pong foi escolhido por aliar diversão e simplicidade em um único projeto. Por ter um sistema de regras simples de ser implementado, foi possível utilizar todos os conhecimentos ensinados ao longo dos últimos três encontros, ao mesmo tempo em que novos conceitos foram introduzidos de forma clara, permitindo que os alunos treinassem o que foi aprendido durante os encontros e pudessem ter uma ideia dos possíveis caminhos a serem seguidos depois daquele dia.

\section{CONSIDERAÇÕES FINAIS}

Este artigo contextualizou o surgimento do grupo Arduemgers ao mostrar que não é uma iniciativa isolada, mas parte de um fenômeno global, o Movimento Maker. Apontou o Critica Making como a metodologia de pesquisa adotada pelo grupo e apresentou a primeira contribuição do grupo à comunidade acadêmica, os workshops de programação e eletrônica básica. A descrição dos encontros apresentou os pontos mais importantes para o aprendizado dos primeiros conceitos e práticas relevantes à prototipagem eletrônica.

A realização de uma série de workshops dentro da Escola de Design teve como objetivo inicial atrair a atenção e mais adeptos para o grupo de estudos. A maior conquista na verdade foi vislumbrar o real potencial de influenciar a comunidade acadêmica quanto a experimentação de diferentes ferramentas de criativas; se tornar um ponto de contato com outros grupos e laboratórios participantes do movimento Maker em Belo Horizonte; estabelecer uma "cultura de desenvolvimento e pesquisa" em um grupo que tem como característica essencial a prática. O que antes era um grupo de estudos em Arduíno hoje se tornou em um grupo de pesquisa em Sistemas Interativos já em processo de registro no CNPq. Os integrantes, dentre eles os autores deste artigo, pretendem manter uma produção constante de artigos; a realização semestral de workshops em programação e prototipagem eletrônica para divulgação, entrada de novos participantes e nivelamento; forte geração de projetos que integrem conceitos de design às possibilidades das novas tendências em tecnologia. 


\section{REFERÊNCIAS}

ANDERSON, C. Makers: The new industrial revolution. New York: Crown. 2012

ARDUINO. Arduino Single-Sided Serial Board (version 3). Disponível na internet por http em: <http://www.arduino.cc/en/Main/ArduinoBoardSerialSingleSided3>. Acesso em 07 maio 2016.

BLIKSTEIN, Paulo; KRANNICH, Dennis. The Makers' Movement and FabLabs in Education: Experiences, Technologies, and Research. IDC 2013, New York, NY, USA. Disponível na internet por http em: <http://dl.acm.org/citation.cfm?id=2485884>. Acesso em 10 maio 2016.

BÜRDEK, Bernhard. Design - História, Teoria e Prática do Design de Produtos. Business, 2010.

CARDOSO, Rafael. Uma Introdução à História do Design. Editora Blücher, São Paulo, 2008.

DOUGHERTY, Dale. The Maker Moviment. Innovations / volume 7, number 3, 2012. http://www.mitpressjournals.org/doi/pdf/10.1162/INOV_a_00135. Acesso em 10 maio 2016.

DOUGHERTY; Dale. The maker mindset. In: HONEY, Margaret; KANTER David (Org.).

Design, Make, Play: Growing the Next Generation of STEM Innovators. Ed, Routledge, NY, USA, 2013. P. 7-11

HALVERSON, Erica; SHERIDAN, Kimberly. The Maker Movement in Education. Harvard Educational Review. Vol. 84, N. 4, 2014. Disponível na internet por http em: <http://lbym.sonoma.edu/i3/sites/default/files/i3/project/Curriculum/Manuals_and_ Reference/Maker_Movement.pdf >. Acesso em 10 maio 2016.

KENT, Steven. The Ultimate History of Video Games: From Pong to Pokemon--The Story Behind the Craze That Touched Our Lives and Changed the World. Prima Pub, New York, 2001.

MARTIN, Lee. The Promise of the Maker Movement for Education. Journal of PreCollege Engineering Education Research (J-PEER). Vol. 5, N. 1, 2015. Disponível na internet por http em:<http://docs.lib.purdue.edu/jpeer/vol5/iss1/4/>. Acesso em 10 maio 2016.

RATTO, Matt. Critical Making: Conceptual and Material Studies in Technology and Social Life. The Information Society, 27:4, 252-260, 2011. Disponível na internet por http em: <http://dx.doi.org/10.1080/01972243.2011.583819>. Acesso em: 12 abr. 2016

RITCHIE, Dennis. The Development of the C Language. Bell Labs/Lucent Technologies, 1999. Disponível na internet por http em: < https://www.belllabs.com/usr/dmr/www/chist.html>. Acesso em 12 abr. 2016. 